\title{
32. PHOSPHORUS GEOCHEMISTRY IN CEARA RISE SEDIMENTS ${ }^{1}$
}

\author{
Margaret L. Delaney² and Linda D. Anderson ${ }^{2}$
}

\begin{abstract}
We applied a sequential extraction technique that distinguishes five sedimentary phosphorus $(\mathrm{P})$ components to sediments from three sites along a water depth transect on the Ceara Rise in the western equatorial Atlantic (Site 925, $3041 \mathrm{~m}$ water depth; Site 926, $3598 \mathrm{~m}$; and Site 929, $4356 \mathrm{~m}$ ). P concentrations in those components that serve to remove reactive P from the oceanic reservoir - adsorbed $\mathrm{P}$, oxide-bound $\mathrm{P}$, authigenic $\mathrm{P}$, and organic $\mathrm{P}$ - account for the majority of sedimentary $\mathrm{P}$ in all sites. Detrital P typically accounts for a small fraction of total P, averaging $4 \%-5 \%$ of total $\mathrm{P}$. Detrital P concentrations are similar at all three sites before about $10 \mathrm{Ma}$, and the two northernmost sites (925 and 929) show large increases (3-5 times) in detrital P concentrations over the past 10 m.y. Organic $\mathrm{P}$, oxide-bound $\mathrm{P}$, and adsorbed $\mathrm{P}$ concentrations account for minor fractions of total reactive $\mathrm{P}$ (typically $9 \%-12 \%, 8 \%-10 \%$, and $7 \%-9 \%$, respectively). $\mathrm{P}$ concentrations in these sedimentary components generally decrease with increasing sample age, with the largest changes in the past $10 \mathrm{~m} . \mathrm{y}$. The decreases in these components are offset by increases in authigenic $\mathrm{P}$ concentration, which constitutes a majority of reactive $\mathrm{P}$ burial. The reorganization of sedimentary $\mathrm{P}$ in the Ceara Rise sites with sediment age is similar to that observed in equatorial Pacific sediments. Neither calcium carbonate contents nor total reactive P concentrations in these sediments correlate with linear sedimentation rates. Reactive $\mathrm{P}$ concentrations do not appear to be directly affected by calcium carbonate dissolution.
\end{abstract}

\section{INTRODUCTION}

The Cenozoic history of the oceanic budget of phosphorus (P), a limiting nutrient for primary productivity on geologic time scales, is not well known (see Delaney and Filippelli [1994] for discussion). Dissolved $P$ enters the ocean by rivers and exits primarily via organic particles transported to the sediment-water interface (Fig. 1). This paper focuses on the burial sinks for removing reactive $\mathrm{P}$ from the oceanic reservoir. A more complete understanding of sedimentary $\mathrm{P}$ geochemistry and its influence on $\mathrm{P}$ accumulation rates in marine sediments would help define how to monitor oceanic $\mathrm{P}$ mass balance changes through time.

Our previous work on $\mathrm{P}$ burial in open-ocean sediments over Cenozoic time scales has concentrated on equatorial Pacific sediments because of the significance of this region in net global new primary productivity and in sediment burial. Geochemical studies on eastern and western equatorial Pacific sediments (from sites from Ocean Drilling Program [ODP] Leg 138, eastern equatorial Pacific, and ODP Leg 130, Ontong-Java Plateau, western equatorial Pacific) have demonstrated that the sedimentary geochemistry of $\mathrm{P}$ in different sites shows similar trends with age. In particular, an authigenic P-rich phase (presumably carbonate fluorapatite) is an important sedimentary sink for reactive $\mathrm{P}$ in open-ocean sediments, and the relative importance of this sink increases with increasing sediment age, serving to retain reactive $P$ in sediments (Filippelli and Delaney, 1995, 1996). However, $\mathrm{P}$ accumulation rate records from these equatorial Pacific sites do not resemble either the increase in continental weathering over the last $40 \mathrm{~m} . \mathrm{y}$. suggested from strontium isotope records or the decrease in relative organic carbon burial rates over the last 15 m.y. indicated by carbon isotope records (Delaney and Filippelli, 1994; Filippelli and Delaney, 1994). Records of P burial rates from other oceans, along with a more complete understanding of the controls on $\mathrm{P}$ accumulation, are needed to better define global oceanic reactive $\mathrm{P}$

${ }^{1}$ Shackleton, N.J., Curry, W.B., Richter, C., and Bralower, T.J., (Eds.), 1997. Proc. ODP, Sci. Results, 154: College Station, TX (Ocean Drilling Program).

${ }^{2}$ Institute of Marine Sciences, University of California, Santa Cruz, CA 95064, U.S.A.delaney@cats.ucsc.edu burial and its relationship to continental weathering and organic carbon budgets.

We investigated the $\mathrm{P}$ concentrations and geochemistry of sediments from three sites along the depth transect drilled in the western equatorial Atlantic on the Ceara Rise (Table 1). These sites share similar surface water productivity, similar sediment inputs, and similar lithology. Calcium carbonate mass accumulation rates generally decrease with increasing site water depth, primarily due to the influence of increasing calcium carbonate dissolution with increasing water depth. Although our ultimate goal is the construction of P accumulation rate records, in this contribution we focus on the influence of sediment characteristics (calcium carbonate content, sedimentation rate) on $\mathrm{P}$ concentrations and geochemistry.

\section{METHODS}

Sediment samples $\left(10 \mathrm{~cm}^{3}\right)$ were taken from Sites 925,926 , and 929. Some samples from Site 926 were splits of the squeeze cakes remaining after interstitial water extraction; previous work has shown good agreement between these different types of samples (Filippelli and Delaney, 1995). Sub-samples (approximately $2.5 \mathrm{~cm}^{3}$ ) of bulk samples were dried overnight at $80^{\circ} \mathrm{C}$ to remove moisture, ground with an agate mortar and pestle, and sieved using a nylon 120-mesh screen $(125 \mu \mathrm{m})$ to prepare a sample having a limited grain-size distribution for subsequent chemical extraction. Ages were assigned to all samples from the biostratigraphic control points used for determining sedimentation rates shipboard (Shipboard Scientific Party, $1995 b, 1995 c, 1995 d)$. Some of our samples were from different holes than those used in the sedimentation rate tables; we used the identifications of these age control points in the biostratigraphy tables to assign depths for the age control points for these holes. Linear sedimentation rates were calculated from age-depth control points and used to interpolate ages.

We applied a sequential extraction technique (Ruttenberg, 1990, 1992), as used in the equatorial Pacific sediment study (Filippelli and Delaney, 1995, 1996), to examine P geochemistry. This chemically isolates $\mathrm{P}$ from five sedimentary components, depending on the dissolution characteristics of the components and on carefully optimized 
Figure 1. The oceanic mass balance of dissolved reactive $\mathrm{P}$. The primary input is the dissolved $\mathrm{P}$ flux from continental weathering carried by rivers to the ocean. The oceanic distribution of dissolved reactive $\mathrm{P}$ is dominated by internal recycling processes, including primary productivity, the associated particle export from the surface ocean, and particle regeneration in the water column, and by ocean circulation. Transport to the sediments is as organic $\mathrm{P}$ in particulate organic matter and as P scavenged by iron-rich hydrothermal particles. Reactive $\mathrm{P}$ burial occurs in different sedimentary forms, and the form in which $\mathrm{P}$ is transported to the sediments is not necessarily the final form in which it is buried. Diagenetic transformations such as the oxidation of organic $\mathrm{P}$ compounds or the precipitation of $\mathrm{P}$ rich authigenic phases can alter one form of sedimentary $\mathrm{P}$ to another after burial.

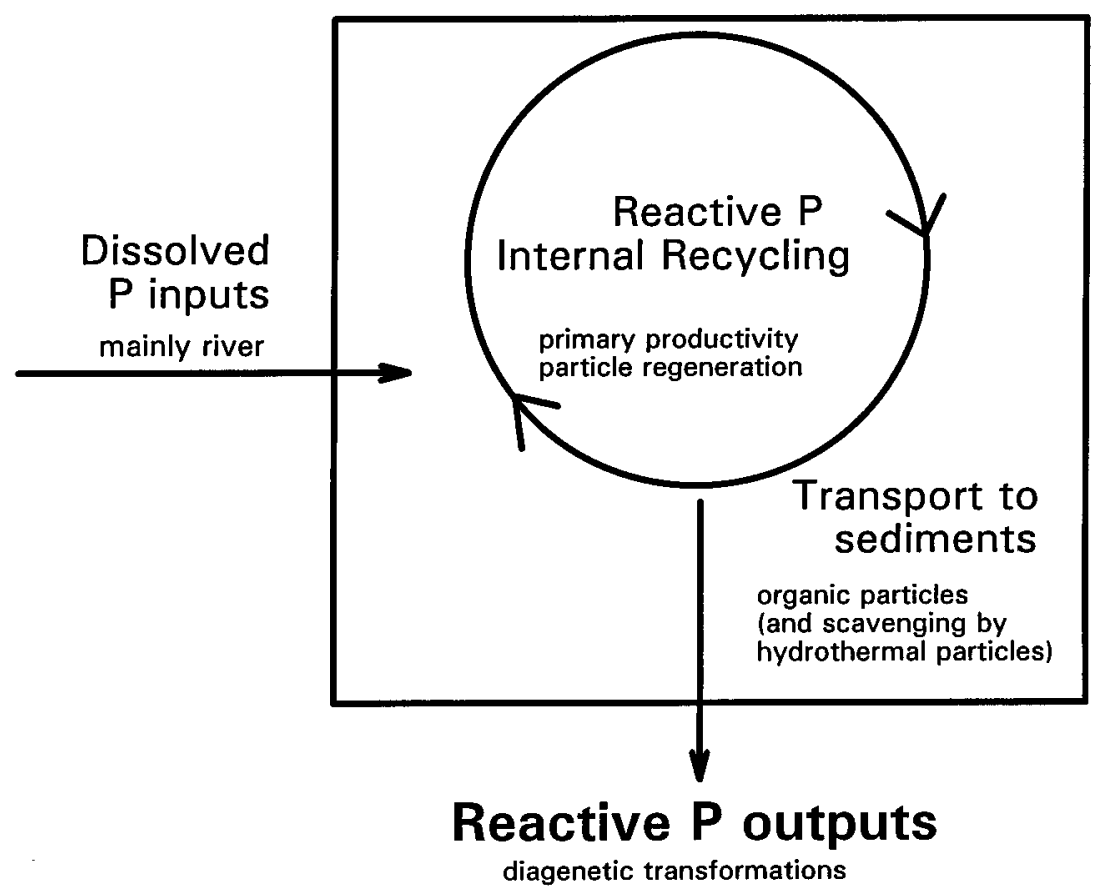

reagent strengths, acid strengths, reaction order, and reaction times (Fig. 2). Briefly, two 0.1-g replicates of each ground sample were placed in new $15 \mathrm{~mL}$ polyethylene centrifuge tubes. During each reaction shown in Figure 2, the centrifuge tubes were shaken on an orbital shaker. After each reaction, samples were centrifuged at 4500 rpm for $12 \mathrm{~min}$, and the supernatants were poured from the centrifuged samples into acid-cleaned polyethylene collection bottles. Supernatants from a reaction step involving multiple treatments were pooled into a single collection bottle. Acid cleaning $\left(1 \mathrm{~N} \mathrm{HCl}\right.$ at $60^{\circ} \mathrm{C}$ for at least $24 \mathrm{hr}$ ), followed by rinsing with glass distilled water (GDW), was used for cleaning all glassware and plasticware; GDW was used for reagent preparation, water washes of the samples, and sample dilutions.

For all steps other than oxide-bound $\mathrm{P}$, the standard ascorbic acid molybdate blue technique (Strickland and Parsons, 1972) was used for developing color. Sample absorbances were measured using a Shimadzu UV-2101PC scanning spectrophotometer with a computer interface and either a low-volume, $10-\mathrm{cm}$ cell or a Sipper 260 autosipper attachment introducing samples to a $1-\mathrm{cm}$ fixed quartz analysis cell. Sample absorbances, zeroed against GDW, were measured at $885 \mathrm{~nm}$ with a slit width of $5 \mathrm{~nm}$. Reagent blanks and standards were prepared to match the matrix of each sample step analyzed. Samples were undiluted for adsorbed $\mathrm{P}$, diluted 1:10 for authigenic $\mathrm{P}$, and diluted 1:5 for detrital and organic $\mathrm{P}$ determinations. The presence of the citrate-dithionite buffer used for oxide-bound $\mathrm{P}$ interferes with the reduction of the molybdate-P complex; therefore, an organic extraction of the complex into isobutanol was used to remove the interfering complex (Watanabe and Olsen, 1962; Ruttenberg, 1992) The extracted complex was reduced with $\mathrm{SnCl}_{2}$, diluted with ethanol, and the absorbance of the colored solution was measured in a 1-cm quartz cell at $730 \mathrm{~nm}$ with a slit width of $5 \mathrm{~nm}$.

Typical analytical detection limits, calculated as three times the standard deviation of the reagent blank, were equivalent to $0.06 \mu \mathrm{mol}$ $\mathrm{P} / \mathrm{g}$ sediment for adsorbed $\mathrm{P}, 0.3$ for oxide-bound $\mathrm{P}, 0.4$ for authigenic P, 0.1 for detrital P, and 0.1 for organic P. Sample absorbances for authigenic $\mathrm{P}$ were always well above the detection limit. Sample absorbances for the other P components ranged from below or near the detection limit to well above the detection limit. Errors are reported in the results section as sample standard deviations for the means of $P$ determinations for each of the five components from replicate samples. Errors are typically larger when concentrations are close to the detection limit for a given component. Relative errors on replicate determinations of individual solutions within a given analytical run were small compared with the mean error on solid replicates. Total reactive $\mathrm{P}$ was calculated as the sum of adsorbed, oxide-bound, authigenic, and organic $\mathrm{P}$, with absolute errors on these sums calculated by the propagation of the absolute errors from individual components.

To evaluate long-term analytical variability, we analyzed two composite samples here termed "consistency standards" (the first, homogenized from splits from a number of samples from Leg 138, Site 844 [Filippelli and Delaney, 1995] with calcium carbonate content of $69.3 \%$, and the second, made for this study, homogenized from splits from samples from Leg 130, Sites 803, 804, and 807, with calcium carbonate content of $89.7 \%$ ). These were run as samples at the beginning and end of each set of samples processed through the extraction sequence. The relative errors (1s) on the long-term means of these consistency standards were relatively high for the four components which constitute relatively small fractions of total P: around $45 \%$ for adsorbed P, $60 \%$ for oxide-bound P, $40 \%$ for detrital P, and $60 \%$ for organic $\mathrm{P}$. The relative errors on the long-term means for authigenic $\mathrm{P}$, the dominant sedimentary component, were $7 \%-9 \%$, with the relative error on means for total $\mathrm{P}=8 \%-10 \%$. The long-term means determined in this study for each P component of the Leg 138 consistency standard agree within error with those from an earlier study (Filippelli and Delaney, 1995); in particular, mean authigenic $\mathrm{P}$ concentrations agree within $4 \%$, and the mean total $\mathrm{P}$ concentrations are within $3 \%$ of each other.

Calcium carbonate contents (reported as weight percent $\mathrm{CaCO}_{3}$ ) were determined on 5- to $10-\mathrm{mg}$ solid aliquots using a UIC, Inc., Coulometrics Model $5012 \mathrm{CO}_{2}$ coulometer. Relative standard deviations on the means for multiple determinations of a pure calcium carbonate standard, for samples run in duplicate within a given analytical run, and for replicate analyses of the two consistency standards over a number of analytical runs were always less than $1 \%$.

\section{RESULTS AND DISCUSSION}

We address the $\mathrm{P}$ geochemistry of these sites (Table 1) and the influence of site and sediment characteristics (Table 2) on these P concentrations. All sites are expected to have similar sedimentary inputs 
Table 1. Sample information and $P$ concentrations (means \pm 1 s standard deviations in $\mu$ mol $P / g$ sediment) for five sedimentary components and for total reactive $P$.

\begin{tabular}{|c|c|c|c|c|c|c|c|c|c|c|}
\hline Hole & Depth & Age & & $\mathrm{CaC}$ & & & concentration & ol P/g sedime & & \\
\hline interval $(\mathrm{cm})$ & (mbsf) & (Ma) & (mbsf/m.y.) & $(\%)$ & Adsorbed & Oxide-bound & Authigenic & Detrital & Organic & Total reactive \\
\hline $925 \mathrm{~B}-3 \mathrm{H}-2,75-77$ & 16.25 & 0.49 & 28.68 & 34.5 & $2.01 \pm 0.15$ & $2.82 \pm 0.56$ & $4.35 \pm 0.26$ & $1.39 \pm 0.27$ & $3.50 \pm 0.39$ & $12.69 \pm 0.75$ \\
\hline $925 \mathrm{D}-4 \mathrm{H}-4,47-49$ & 35.97 & 1.18 & 27.87 & 49.8 & $2.29 \pm 0.06$ & $1.34 \pm 0.06$ & $8.93 \pm 0.78$ & $0.83 \pm 0.15$ & $1.14 \pm 0.22$ & $13.71 \pm 0.82$ \\
\hline $925 \mathrm{D}-6 \mathrm{H}-3,48-50$ & 53.48 & 1.80 & 27.87 & 54.5 & $1.45 \pm 0.19$ & $2.38 \pm 1.23$ & $4.97 \pm 0.92$ & $2.46 \pm 0.16$ & $2.47 \pm 0.19$ & $11.27 \pm 1.56$ \\
\hline $925 \mathrm{D}-8 \mathrm{H}-3,49-51$ & 72.49 & 2.45 & 29.86 & 43.9 & $1.60 \pm 0.01$ & $1.36 \pm 0.43$ & $4.53 \pm 0.54$ & $1.12 \pm 0.06$ & $2.86 \pm 0.36$ & $10.34 \pm 0.78$ \\
\hline $925 \mathrm{D}-10 \mathrm{H}-1,59-61$ & 88.59 & 2.99 & 29.86 & 57.0 & $1.89 \pm 0.94$ & $0.92 \pm 0.07$ & $9.57 \pm 1.27$ & $0.82 \pm 0.09$ & $0.96 \pm 0.09$ & $13.33 \pm 1.58$ \\
\hline $925 \mathrm{D}-12 \mathrm{H}-2,48-50$ & 108.98 & 3.67 & 29.86 & 54.4 & $0.93 \pm 0.01$ & $2.64 \pm 0.24$ & $4.28 \pm 0.37$ & $1.81 \pm 0.05$ & $2.48 \pm 0.46$ & $10.33 \pm 0.64$ \\
\hline $925 \mathrm{D}-25 \mathrm{H}-3,49-51$ & 233.99 & 8.89 & 23.72 & 79.5 & $0.26 \pm 0.01$ & $1.38 \pm 0.18$ & $9.36 \pm 0.63$ & $0.61 \pm 0.23$ & $1.31 \pm 0.21$ & $12.31 \pm 0.69$ \\
\hline $925 \mathrm{D}-29 \mathrm{H}-2,49-51$ & 279.99 & $\begin{array}{r}.06 \\
11.30\end{array}$ & 15.83 & 64.4 & $0.37 \pm 0.13$ & $1.91 \pm 0.70$ & $6.72 \pm 1.51$ & $1.94 \pm 0.10$ & $1.67 \pm 1.00$ & $10.68 \pm 1.95$ \\
\hline $925 \mathrm{~B}-31 \mathrm{H}-3,75-77$ & 283.75 & 11.75 & 13.32 & 78.8 & $0.67 \pm 0.04$ & $0.39 \pm 0.22$ & $8.93 \pm 0.26$ & $0.31 \pm 0.13$ & $1.01 \pm 0.10$ & $11.00 \pm 0.36$ \\
\hline $925 \mathrm{~B}-33 \mathrm{H}-2,75-77$ & 301.25 & 13.06 & 13.32 & 68.6 & 1.29 & $0.43 \pm 0.14$ & $10.53 \pm 0.50$ & $0.46 \pm 0.16$ & $0.58 \pm 0.14$ & $12.84 \pm 0.54$ \\
\hline $925 \mathrm{~A}-4 \mathrm{R}-7,35-37$ & 323.05 & 14.88 & 10.16 & 75.7 & $0.49 \pm 0.11$ & $0.37 \pm 0.09$ & $8.68 \pm 0.94$ & $0.25 \pm 0.04$ & $1.09 \pm 0.13$ & $10.62 \pm 0.96$ \\
\hline $925 \mathrm{D}-36 \mathrm{H}-2,49-51$ & 336.99 & 15.97 & 49.38 & 67.9 & $0.20 \pm 0.04$ & $1.52 \pm 0.32$ & $6.57 \pm 0.46$ & $0.32 \pm 0.10$ & $1.82 \pm 0.12$ & $10.12 \pm 0.57$ \\
\hline $925 \mathrm{~A}-15 \mathrm{R}-1,50-52$ & 420.20 & 19.04 & 15.31 & 80.1 & $1.32 \pm 0.20$ & $0.38 \pm 0.33$ & $9.08 \pm 0.48$ & $0.13 \pm 0.05$ & $0.27 \pm 0.01$ & $11.04 \pm 0.61$ \\
\hline $925 \mathrm{~A}-17 \mathrm{R}-1,50-52$ & 439.40 & 20.29 & 15.31 & 75.0 & $0.21 \pm 0.12$ & $0.94 \pm 0.23$ & $9.07 \pm 0.67$ & $0.37 \pm 0.10$ & $1.29 \pm 0.07$ & $11.51 \pm 0.72$ \\
\hline $925 \mathrm{~A}-19 \mathrm{R}-2,50-52$ & 458.77 & 21.56 & 15.31 & 66.5 & $0.75 \pm 0.09$ & $0.27 \pm 0.03$ & $10.96 \pm 0.36$ & $0.31 \pm 0.07$ & $1.13 \pm 0.11$ & $13.12 \pm 0.39$ \\
\hline $925 \mathrm{~A}-20 \mathrm{R}-7,50-52$ & 477.30 & 22.53 & 19.23 & 80.1 & $1.06 \pm 0.03$ & $0.37 \pm 0.10$ & $9.32 \pm 0.44$ & $0.23 \pm 0.03$ & $0.29 \pm 0.06$ & $11.04 \pm 0.46$ \\
\hline $925 \mathrm{~A}-23 \mathrm{R}-1,50-52$ & 497.20 & 23.57 & 19.23 & 71.3 & $0.11 \pm 0.07$ & $1.43 \pm 0.32$ & $11.94 \pm 0.90$ & $0.34 \pm 0.02$ & $1.12 \pm 0.43$ & $14.61 \pm 1.05$ \\
\hline $925 \mathrm{~A}-26 \mathrm{R}-1,30-32$ & 525.90 & 25.04 & 19.51 & 63.1 & $0.56 \pm 0.04$ & $0.49 \pm 0.20$ & $8.81 \pm 0.53$ & $0.53 \pm 0.06$ & $1.08 \pm 0.10$ & $10.94 \pm 0.58$ \\
\hline $925 \mathrm{~A}-27 \mathrm{R}-3,50-52$ & 538.80 & 25.70 & 19.51 & 68.4 & $0.65 \pm 0.20$ & $0.25 \pm 0.09$ & $8.83 \pm 0.57$ & $0.27 \pm 0.05$ & $0.64 \pm 0.07$ & $10.37 \pm 0.62$ \\
\hline $925 \mathrm{~A}-29 \mathrm{R}-1,50-52$ & 555.10 & 26.53 & 19.51 & 65.4 & $0.45 \pm 0.04$ & $0.25 \pm 0.10$ & $12.39 \pm 0.48$ & $0.61 \pm 0.15$ & $1.15 \pm 0.12$ & $14.24 \pm 0.50$ \\
\hline $925 \mathrm{~A}-31 \mathrm{R}-2,50-52$ & 575.80 & 27.60 & 19.51 & $\begin{array}{l}0.4 \\
72.4\end{array}$ & $0.18 \pm 0.10$ & $1.04 \pm 0.10$ & $10.55 \pm 1.55$ & $0.39 \pm 0.04$ & $1.11 \pm 0.30$ & $12.88 \pm 1.59$ \\
\hline $925 \mathrm{~A}-33 \mathrm{R}-5,50-52$ & 599.60 & 28.65 & 39.93 & 80.6 & $0.37 \pm 0.05$ & $0.90 \pm 0.01$ & $8.30 \pm 0.13$ & $0.26 \pm 0.05$ & $0.81 \pm 0.22$ & $10.37 \pm 0.26$ \\
\hline $925 \mathrm{~A}-35 \mathrm{R}-3,50-52$ & 615.90 & 29.06 & 39.93 & 84.2 & $0.41 \pm 0.12$ & $0.93 \pm 0.90$ & $6.38 \pm 0.19$ & $0.12 \pm 0.07$ & $0.79 \pm 0.06$ & $8.51 \pm 0.93$ \\
\hline $925 \mathrm{~A}-38 \mathrm{R}-1,50-52$ & 641.90 & 29.71 & 37.98 & 79.8 & $0.07 \pm 0.11$ & $1.02 \pm 0.20$ & $12.68 \pm 1.39$ & $0.84 \pm 0.53$ & $1.10 \pm 0.21$ & $14.87 \pm 1.42$ \\
\hline $925 \mathrm{~A}-39 \mathrm{R}-7,50-52$ & 660.13 & 30.19 & 37.98 & 81.0 & $0.31 \pm 0.03$ & $0.22 \pm 0.21$ & $7.72 \pm 0.43$ & $0.10 \pm 0.02$ & $0.71 \pm 0.21$ & $8.96 \pm 0.53$ \\
\hline $925 \mathrm{~A}-42 \mathrm{R}-1,52-54$ & 680.62 & 30.73 & 37.98 & 76.5 & $1.01 \pm 0.16$ & $0.31 \pm 0.11$ & $8.53 \pm 0.35$ & $0.17 \pm 0.09$ & $0.43 \pm 0.06$ & $10.28 \pm 0.40$ \\
\hline $925 \mathrm{~A}-45 \mathrm{R}-6,50-52$ & $\begin{array}{l}0807.32 \\
707.30\end{array}$ & 31.443 & $\begin{array}{r}7.90 \\
7.98\end{array}$ & 74.1 & $1.08 \pm 0.98$ & $0.40 \pm 0.05$ & $11.04 \pm 0.54$ & $0.07 \pm 0.20$ & $0.35 \pm 0.04$ & $12.87 \pm 1.12$ \\
\hline $925 \mathrm{~A}-47 \mathrm{R}-3,50-52$ & 721.80 & 31.82 & 37.98 & 61.9 & $0.24 \pm 0.08$ & $0.79 \pm 0.45$ & $8.59 \pm 0.91$ & $0.37 \pm 0.06$ & $1.25 \pm 0.31$ & $10.87 \pm 1.06$ \\
\hline $925 \mathrm{~A}-49 \mathrm{R}-2,49-51$ & 739.59 & 32.29 & 37.98 & 62.2 & $0.63 \pm 0.05$ & $0.32 \pm 0.04$ & $11.37 \pm 0.40$ & $0.28 \pm 0.02$ & $1.09 \pm 0.11$ & $13.42 \pm 0.41$ \\
\hline $925 \mathrm{~A}-52 \mathrm{R}-3,50-52$ & 760.50 & 32.95 & 20.40 & 62.2 & $1.55 \pm 0.46$ & $0.22 \pm 0.04$ & $9.28 \pm 0.43$ & $0.42 \pm 0.15$ & $0.45 \pm 0.04$ & $11.49 \pm 0.63$ \\
\hline $925 \mathrm{~A}-54 \mathrm{R}-3,50-52$ & 779.80 & 33.94 & 16.16 & 63.7 & $1.32 \pm 0.20$ & $0.29 \pm 0.04$ & $9.99 \pm 0.62$ & $0.30 \pm 0.12$ & $0.63 \pm 0.10$ & $12.23 \pm 0.67$ \\
\hline $925 \mathrm{~A}-56 \mathrm{R}-3,50-52$ & 799.10 & 35.13 & 16.16 & 59.4 & $1.40 \pm 0.07$ & $0.22 \pm 0.08$ & $11.76 \pm 0.79$ & $0.44 \pm 0.11$ & $0.55 \pm 0.06$ & $13.93 \pm 0.80$ \\
\hline $925 \mathrm{~A}-58 \mathrm{R}-4,50-52$ & 819.80 & 36.41 & 16.16 & 66.4 & $0.05 \pm 0.04$ & $0.90 \pm 0.23$ & $10.73 \pm 0.47$ & $0.28 \pm 0.04$ & $0.96 \pm 0.26$ & $12.64 \pm 0.59$ \\
\hline $925 \mathrm{~A}-60 \mathrm{R}-3,50-52$ & 837.70 & 37.52 & 16 & 56.5 & $0.69 \pm 0.06$ & $0.89 \pm 0.70$ & $11.88 \pm 0.41$ & $0.61 \pm 0.06$ & $1.28 \pm 0.38$ & $14.74 \pm 0.90$ \\
\hline $925 \mathrm{~A}-62 \mathrm{R}-5,50-52$ & 860.00 & 38.90 & 16.16 & 65.2 & $1.58 \pm 0.47$ & $0.35 \pm 0.11$ & $10.65 \pm 0.85$ & $0.37 \pm 0.13$ & $0.39 \pm 0.08$ & $12.96 \pm 0.98$ \\
\hline $925 \mathrm{~A}-64 \mathrm{R}-5,50-52$ & 879.30 & 40.10 & 16.16 & 64.2 & $0.58 \pm 0.05$ & $0.59 \pm 0.25$ & $10.22 \pm 2.34$ & $0.22 \pm 0.07$ & $0.67 \pm 0.31$ & $12.06 \pm 2.37$ \\
\hline $925 \mathrm{~A}-66 \mathrm{R}-1,50-52$ & 892.30 & 40.89 & 16.32 & 55.5 & $1.46 \pm 0.39$ & $0.33 \pm 0.04$ & $10.93 \pm 0.61$ & $0.62 \pm 0.16$ & $0.57 \pm 0.10$ & $13.29 \pm 0.73$ \\
\hline $925 \mathrm{~A}-69 \mathrm{R}-1,50-52$ & 921.20 & 42.66 & 16.32 & 75.9 & $0.04 \pm 0.04$ & $1.49 \pm 0.14$ & $8.41 \pm 0.31$ & $0.12 \pm 0.08$ & $1.57 \pm 0.22$ & $11.51 \pm 0.41$ \\
\hline $926 \mathrm{C}-2 \mathrm{H}-4,52-54$ & 15.02 & 0.49 & 29.30 & 54.8 & $2.38 \pm 0.12$ & $3.78 \pm 0.07$ & $6.70 \pm 0.47$ & $0.65 \pm 0.04$ & $1.65 \pm 0.04$ & $14.52 \pm 0.49$ \\
\hline $926 \mathrm{C}-6 \mathrm{l}$ & 54.50 & 1.94 & 29.30 & 48.4 & $1.80 \pm 0.22$ & $1.68 \pm 0.16$ & $4.93 \pm 1.06$ & $0.56 \pm 0.07$ & $1.48 \pm 0.11$ & $9.89 \pm 1.10$ \\
\hline $926 \mathrm{C}-8 \mathrm{H}-5,59-61$ & 73.59 & 2.62 & 27.94 & 49.5 & $1.45 \pm 0.07$ & $2.24 \pm 0.17$ & $6.86 \pm 0.97$ & $0.67 \pm 0.05$ & $1.47 \pm 0.19$ & $12.03 \pm 1.01$ \\
\hline $926 \mathrm{C}-10 \mathrm{H}-4,50-52$ & 91.00 & 3.24 & 27.94 & 69.6 & $1.31 \pm 0.04$ & $0.79 \pm 0.14$ & $7.53 \pm 0.66$ & $0.30 \pm 0.03$ & $0.88 \pm 0.12$ & $10.52 \pm 0.69$ \\
\hline $926 \mathrm{C}-12 \mathrm{H}-2,50-52$ & 107.00 & 3.82 & 23.55 & 55.9 & $1.31 \pm 0.05$ & $1.09 \pm 0.17$ & $6.16 \pm 0.38$ & $0.47 \pm 0.02$ & $1.25 \pm 0.12$ & $9.82 \pm 0.44$ \\
\hline $926 \mathrm{~A}-14 \mathrm{H}-5,49-51$ & 124.49 & 4.55 & 23.79 & 69.2 & $1.16 \pm 0.04$ & $1.01 \pm 0.15$ & $5.98 \pm 0.52$ & $0.33 \pm 0.04$ & $1.04 \pm 0.30$ & $9.19 \pm 0.62$ \\
\hline $926 \mathrm{~A}-16 \mathrm{H}-4,50-52$ & 142.00 & 5.28 & 23.79 & 69.0 & $0.84 \pm 0.25$ & $1.11 \pm 0.19$ & $6.25 \pm 0.44$ & $0.31 \pm 0.06$ & $1.47 \pm 0.73$ & $9.67 \pm 0.91$ \\
\hline $926 \mathrm{C}-17 \mathrm{H}-5,50-52$ & 159.00 & 6.08 & 17.94 & 50.3 & $1.31 \pm 0.06$ & $2.74 \pm 0.20$ & $5.72 \pm 0.49$ & $0.57 \pm 0.15$ & $1.64 \pm 0.36$ & $11.41 \pm 0.65$ \\
\hline $926 \mathrm{~A}-21 \mathrm{H}-3,145-150$ & 188.95 & 8.01 & 15.40 & 72.8 & $0.72 \pm 0.19$ & $0.21 \pm 0.14$ & $5.98 \pm 0.51$ & $0.26 \pm 0.02$ & $0.67 \pm 0.09$ & $7.58 \pm 0.57$ \\
\hline $926 \mathrm{C}-22 \mathrm{H}-2,50-52$ & 202.00 & 8.47 & 18.39 & 72.3 & $0.83 \pm 0.12$ & $0.71 \pm 0.22$ & $5.70 \pm 0.39$ & $0.23 \pm 0.01$ & $0.95 \pm 0.39$ & $8.19 \pm 0.60$ \\
\hline $926 \mathrm{~A}-24 \mathrm{H}-3,145-150$ & 217.45 & 9.76 & 10.63 & 64.1 & $0.74 \pm 0.02$ & $1.39 \pm 1.06$ & $7.17 \pm 2.61$ & $0.16 \pm 0.00$ & $1.13 \pm 0.07$ & $10.42 \pm 2.82$ \\
\hline $926 \mathrm{~A}-27 \mathrm{H}-3,145-150$ & 245.95 & 12.24 & 14.50 & 59.4 & $0.45 \pm 0.08$ & $0.60 \pm 0.18$ & $7.19 \pm 0.70$ & $0.32 \pm 0.02$ & $1.16 \pm 0.18$ & $9.39 \pm 0.75$ \\
\hline $926 \mathrm{~A}-30 \mathrm{H}-3,145-150$ & 274.45 & 14.35 & 11.73 & 70.2 & $0.42 \pm 0.02$ & $0.32 \pm 0.31$ & $10.59 \pm 0.35$ & $0.90 \pm 0.07$ & $0.64 \pm 0.05$ & $11.98 \pm 0.48$ \\
\hline $926 \mathrm{~A}-33 \mathrm{H}-3,145-150$ & 302.95 & 16.78 & 11.73 & 63.9 & $0.51 \pm 0.08$ & 0.41 & $7.47 \pm 0.39$ & $0.12 \pm 0.01$ & $0.55 \pm 0.09$ & $8.94 \pm 0.41$ \\
\hline $925 \mathrm{~B}-36 \mathrm{X}-2,140-150$ & 329.40 & 18.60 & 21.98 & 74.1 & & $0.59 \pm 0.21$ & $10.04 \pm 0.87$ & $0.58 \pm 0.73$ & $0.44 \pm 0.02$ & $11.07 \pm 0.89$ \\
\hline $925 \mathrm{~B}-39 \mathrm{X}-3,140-150$ & 359.90 & 19.93 & 28.08 & 77.0 & $0.38 \pm 0.07$ & $0.23 \pm 0.17$ & $6.45 \pm 0.61$ & $0.19 \pm 0.02$ & $0.40 \pm 0.04$ & $7.47 \pm 0.64$ \\
\hline $925 \mathrm{~B}-42 \mathrm{X}-3,140-150$ & 388.50 & 20.94 & 28.08 & 66.4 & $0.55 \pm 0.08$ & $0.20 \pm 0.08$ & $7.34 \pm 0.47$ & $0.20 \pm 0.01$ & $0.54 \pm 0.09$ & $8.64 \pm 0.49$ \\
\hline $925 \mathrm{~B}-45 \mathrm{X}-3,140-150$ & 417.50 & 21.98 & 28.08 & 81.3 & $0.44 \pm 0.03$ & $0.84 \pm 0.35$ & $7.77 \pm 0.24$ & BDL & $0.34 \pm 0.27$ & $9.37 \pm 0.50$ \\
\hline $925 \mathrm{~B}-48 \mathrm{X}-3,140-150$ & 446.50 & 23.01 & 28.08 & 66.7 & $0.44 \pm 0.04$ & $0.48 \pm 0.50$ & $12.17 \pm 0.69$ & $0.10 \pm 0.01$ & $0.53 \pm 0.06$ & $13.61 \pm 0.85$ \\
\hline $925 \mathrm{~B}-51 \mathrm{X}-3,135-150$ & 475.35 & 24.12 & 22.44 & 58.4 & $0.36 \pm 0.05$ & $0.84 \pm 0.30$ & $7.68 \pm 0.48$ & $0.53 \pm 0.04$ & $0.62 \pm 0.04$ & $9.49 \pm 0.57$ \\
\hline $925 \mathrm{~B}-54 \mathrm{X}-4,135-150$ & 505.75 & 25.48 & 22.44 & 60.1 & $0.61 \pm 0.22$ & $0.26 \pm 0.06$ & $9.68 \pm 0.52$ & $0.38 \pm 0.13$ & $0.75 \pm 0.05$ & $11.29 \pm 0.57$ \\
\hline $925 \mathrm{~B}-57 \mathrm{X}-4,135-150$ & 534.75 & 26.77 & 22.44 & 49.1 & $0.40 \pm 0.03$ & $0.19 \pm 0.22$ & $7.77 \pm 0.17$ & $0.36 \pm 0.04$ & $1.16 \pm 0.10$ & $9.52 \pm 0.29$ \\
\hline $925 \mathrm{~B}-60 \mathrm{X}-3,135-150$ & 562.25 & 28.00 & 22.44 & 73.9 & $0.38 \pm 0.06$ & $0.45 \pm 0.01$ & $6.90 \pm 0.28$ & $0.07 \pm 0.02$ & $0.37 \pm 0.05$ & $8.09 \pm 0.29$ \\
\hline $925 \mathrm{~B}-63 \mathrm{X}-3,135-150$ & 591.25 & 29.31 & 21.81 & 73.3 & $0.79 \pm 0.31$ & $0.33 \pm 0.04$ & $9.14 \pm 0.54$ & $0.56 \pm 0.37$ & $0.40 \pm 0.06$ & $10.66 \pm 0.63$ \\
\hline $929 \mathrm{~A}-2 \mathrm{H}-4,145-150$ & 10.45 & 0.28 & 37.07 & 48.4 & $1.72 \pm 0.07$ & $153+030$ & $12.44 \pm 1.89$ & $1.30 \pm 0.14$ & $1.55 \pm 0.15$ & $17.24 \pm 1.92$ \\
\hline & & & 2 & & 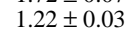 & $3.26 \pm 1.00$ & & 2 & 4.2 & $14.02 \pm 1.16$ \\
\hline $929 \mathrm{~A}-9 \mathrm{H}-3,145-150$ & 75.45 & 2.58 & 25.44 & 31.1 & $1.00 \pm 0.07$ & $0.67 \pm 0.46$ & $7.14 \pm 0.32$ & $2.33 \pm 0.07$ & $1.52 \pm 0.18$ & $10.33 \pm 0.59$ \\
\hline $929 \mathrm{~A}-12 \mathrm{H}-3,145-150$ & 103.95 & 3.70 & 25.44 & 30.7 & $2.69 \pm 0.28$ & $1.19 \pm 0.13$ & $6.15 \pm 0.49$ & $1.17 \pm 0.08$ & $2.21 \pm 0.64$ & $12.23 \pm 0.86$ \\
\hline $929 \mathrm{~A}-15 \mathrm{H}-2,50-52$ & 130.00 & 5.00 & 19.71 & 27.5 & $0.94 \pm 0.19$ & $3.26 \pm 0.47$ & $8.25 \pm 0.51$ & $2.59 \pm 0.39$ & $4.04 \pm 0.72$ & $16.50 \pm 1.02$ \\
\hline$-15 \mathrm{H}-3,145-150$ & 13 & 5.21 & 9 & 3 & 2. & \pm 0.06 & 6.61 & $0.91 \pm 0.23$ & $1.49 \pm 0.13$ & \pm 0.54 \\
\hline $929 \mathrm{~B}-17 \mathrm{X}-6,50-52$ & 160.20 & 6.75 & 26.8 & 41.0 & $1.48 \pm 0.14$ & $1.94 \pm 0.61$ & $5.91 \pm 0.36$ & $1.10 \pm 0.07$ & $3.42 \pm 0.49$ & $12.75 \pm 0.87$ \\
\hline $929 \mathrm{~A}-20 \mathrm{X}-3,50-52$ & 179.60 & 7.41 & 26.53 & 51.6 & $0.92 \pm 0.22$ & $1.94 \pm 0.59$ & $7.29 \pm 0.46$ & $1.32 \pm 0.50$ & $2.20 \pm 1.95$ & $12.35 \pm 2.11$ \\
\hline $929 \mathrm{~A}-23 \mathrm{X}-1,50-52$ & 205.60 & 8.39 & 26.53 & 47.0 & $0.48 \pm 0.14$ & $2.42 \pm 0.78$ & $8.98 \pm 0.46$ & $0.94 \pm 0.64$ & $2.40 \pm 0.23$ & $14.28 \pm 0.95$ \\
\hline $929 \mathrm{~A}-24 \mathrm{X}-3,140-150$ & 218.80 & 13.38 & $\begin{array}{r}30.53 \\
3.24\end{array}$ & $\begin{array}{r}4.0 \\
4.8\end{array}$ & $2.14 \pm 0.72$ & $0.59 \pm 0.08$ & $\begin{array}{r}0.90 \pm 0.40 \\
15.66 \pm 0.80\end{array}$ & $0.98 \pm 0.12$ & $2.68 \pm 0.31$ & $21.07 \pm 1.12$ \\
\hline $929 \mathrm{~A}-26 \mathrm{X}-3,50-52$ & 237.10 & 16.93 & 9.28 & 30.4 & $1.06 \pm 0.10$ & $1.67 \pm 1.48$ & $11.89 \pm 0.25$ & $0.49 \pm 0.04$ & $2.91 \pm 0.14$ & $17.52 \pm 1.52$ \\
\hline $3,50-52$ & 25 & & 7 & 5 & & 1 & 8. & 06 & $\begin{array}{l}0.14 \\
\pm 0.56\end{array}$ & 12 \\
\hline $3,50-52$ & 275.40 & 21.58 & 7.75 & 45.6 & $1.08 \pm 0.10$ & $2.35 \pm 2.45$ & $11.10 \pm 0.41$ & $0.47 \pm 0.04$ & $1.88 \pm 0.10$ & $16.41 \pm 2.49$ \\
\hline $929 \mathrm{~A}-37 \mathrm{X}-3,50-52$ & 343.20 & 24.82 & 17.74 & 65.7 & $0.14 \pm 0.03$ & $1.26 \pm 0.26$ & $8.95 \pm 0.37$ & $0.39 \pm 0.10$ & $1.31 \pm 0.31$ & $11.67 \pm 0.54$ \\
\hline $929 \mathrm{~A}-39 \mathrm{X}-3,80-83$ & 362.81 & 25.92 & 17.74 & 69.5 & $0.61 \pm 0.04$ & $2.49 \pm 3.98$ & $8.04 \pm 0.39$ & $0.29 \pm 0.06$ & $1.12 \pm 0.12$ & $12.25 \pm 4.00$ \\
\hline $929 A-41 X-3,74-76$ & 381.84 & 27.00 & 17.74 & 27.0 & $0.80 \pm 0.12$ & $0.75 \pm 0.40$ & $12.03 \pm 0.25$ & $0.43 \pm 0.13$ & $1.21 \pm 0.08$ & $14.78 \pm 0.49$ \\
\hline $3,58-60$ & 400.9 & 28.07 & 17.74 & 68.4 & $0.73 \pm 0.05$ & $0.57 \pm 0.16$ & $8.86 \pm 0.33$ & & $1.17 \pm 0.11$ & \\
\hline $3,100-102$ & 42 & 29. & 7 & 6 & 0 & $0.98 \pm 0.53$ & $11.62 \pm 0.99$ & & $1.32 \pm$ & $14.16 \pm 1.19$ \\
\hline $929 \mathrm{~A}-47 \mathrm{X}-3,52-54$ & 439.62 & 30.25 & 17.74 & 70.5 & $0.55 \pm 0.09$ & $0.65 \pm 0.41$ & $9.30 \pm 0.03$ & $0.19 \pm 0.04$ & $1.00 \pm 0.07$ & $11.51 \pm 0.43$ \\
\hline $929 \mathrm{~A}-49 \mathrm{X}-3,52-54$ & 458.82 & 31.33 & 17.81 & 65.4 & $0.56 \pm 0.10$ & $0.86 \pm 0.97$ & $9.03 \pm 0.04$ & $0.23 \pm 0.07$ & $1.09 \pm 0.11$ & $11.54 \pm 0.99$ \\
\hline $929 \mathrm{~A}-50 \mathrm{X}-3,135-150$ & 469.35 & 31.92 & 17.81 & 59.4 & $2.19 \pm 0.31$ & $0.40 \pm 0.09$ & $9.84 \pm 0.66$ & $0.22 \pm 0.12$ & $0.69 \pm 0.28$ & $13.13 \pm 0.78$ \\
\hline $929 \mathrm{~A}-51 \mathrm{X}-3,52-54$ & 478.12 & 32.41 & 17.81 & 62.0 & $0.18 \pm 0.23$ & $1.15 \pm 0.31$ & $7.21 \pm 0.33$ & $0.36 \pm 0.15$ & $1.18 \pm 0.50$ & $9.71 \pm 0.71$ \\
\hline$-2,135-150$ & 496.35 & 33.44 & 17.8 & 47.0 & \pm 0.20 & $0.37 \pm 0.17$ & $8.73 \pm 0.38$ & $0.30 \pm 0.14$ & $0.88 \pm 0.10$ & $11.48 \pm 0.48$ \\
\hline $929 \mathrm{E}-6 \mathrm{R}-1,130-150$ & 521.30 & 34.84 & 17.81 & 66.6 & $0.41 \pm 0.08$ & $0.33 \pm 0.17$ & $7.39 \pm 0.48$ & $0.17 \pm 0.01$ & $0.37 \pm 0.07$ & $8.50 \pm 0.52$ \\
\hline
\end{tabular}


Table 1 (continued).

\begin{tabular}{|c|c|c|c|c|c|c|c|c|c|c|}
\hline \multirow{2}{*}{$\begin{array}{l}\text { Hole, core, section, } \\
\text { interval }(\mathrm{cm})\end{array}$} & \multirow{2}{*}{$\begin{array}{l}\text { Depth } \\
\text { (mbsf) }\end{array}$} & \multirow{2}{*}{$\begin{array}{l}\text { Age } \\
(\mathrm{Ma})\end{array}$} & \multirow{2}{*}{$\begin{array}{c}\text { LSR } \\
\text { (mbsf/m.y.) }\end{array}$} & \multirow{2}{*}{$\begin{array}{c}\mathrm{CaCO}_{3} \\
(\%)\end{array}$} & \multicolumn{6}{|c|}{$\mathrm{P}$ concentration $(\mu \mathrm{mol} \mathrm{P} / \mathrm{g}$ sediment $)$} \\
\hline & & & & & Adsorbed & Oxide-bound & Authigenic & Detrital & Organic & Total reactive \\
\hline 929E-10R-5, 130-150 & 565.60 & 37.83 & 5.53 & 60.9 & $0.47 \pm 0.02$ & $0.67 \pm 0.26$ & $9.72 \pm 0.78$ & $0.09 \pm 0.0$ & $10.49 \pm 0.05$ & $11.35 \pm 0.82$ \\
\hline $929 \mathrm{E}-14 \mathrm{R}-3,130-150$ & 601.20 & 41.69 & 16.53 & 57.2 & $0.54 \pm 0.11$ & $2.13 \pm 0.66$ & $10.46 \pm 0.77$ & BDL & $0.46 \pm 0.09$ & $13.57 \pm 1.02$ \\
\hline $929 \mathrm{E}-19 \mathrm{R}-1,130-150$ & 646.30 & 46.78 & 22.61 & 59.9 & $0.40 \pm 0.16$ & $0.42 \pm 0.23$ & $6.80 \pm 0.68$ & $0.08 \pm 0.01$ & $0.32 \pm 0.05$ & $7.94 \pm 0.73$ \\
\hline $929 \mathrm{E}-23 \mathrm{R}-1,130-150$ & 684.50 & 48.86 & 13.50 & 20.6 & $0.53 \pm 0.09$ & $1.28 \pm 0.74$ & $6.04 \pm 0.30$ & $0.09 \pm 0.01$ & $1.36 \pm 0.02$ & $9.22 \pm 0.80$ \\
\hline $929 \mathrm{E}-28 \mathrm{R}-3,130-150$ & 735.90 & 52.67 & 13.50 & 30.9 & $0.67 \pm 0.10$ & $0.14 \pm 0.17$ & $6.25 \pm 0.37$ & $0.17 \pm 0.02$ & $0.69 \pm 0.12$ & $7.75 \pm 0.43$ \\
\hline 929E-33R-2, $128-148$ & 782.68 & 55.21 & 20.06 & 50.8 & $0.61 \pm 0.18$ & $2.28 \pm 0.50$ & $7.82 \pm 0.19$ & BDL & $0.37 \pm 0.021$ & $1.09 \pm 0.57$ \\
\hline
\end{tabular}

Notes: $\mathrm{LSR}=$ linear sedimentation rate. $\mathrm{BDL}=$ value below analytical detection limit. Blank entry = value not determined; when given for standard deviation, this indicates that only one replicate yielded a measurement for that component. Total reactive P is calculated as the sum of adsorbed, oxide-bound, authigenic, and organic P. The absolute error on this sum is calculated by propagation of the absolute errors on the individual components.

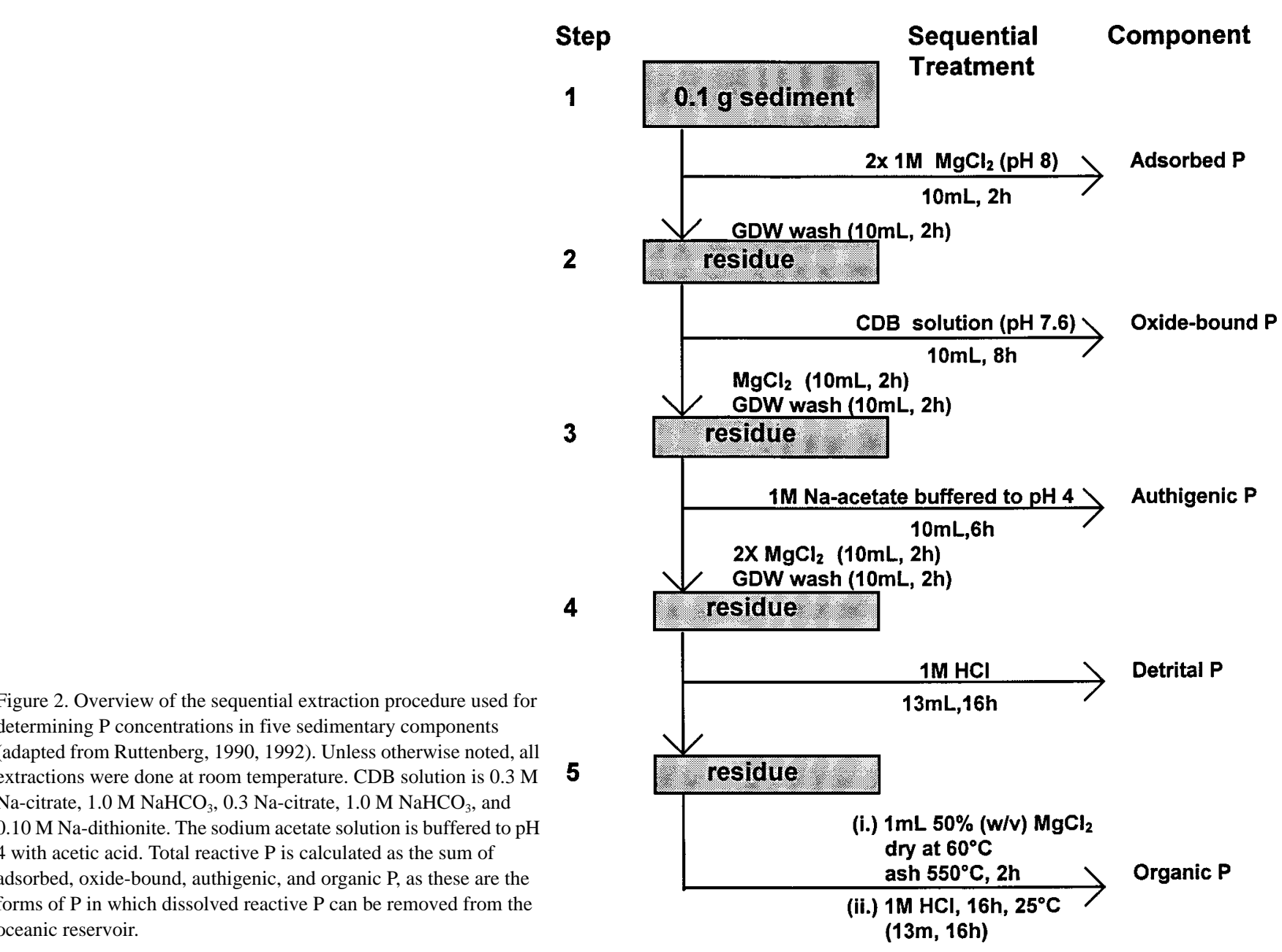

through time. The primary differences between sites are due to the increasing intensity of calcium carbonate dissolution with water depth, as well as the variations in the dissolution-depth relationship through time. Calcium carbonate contents have similar trends for the past 30 m.y. for the two shallowest sites (Sites 925 and 926; Tables 1, 2), with averages around $70 \%$ from 10 to $30 \mathrm{Ma}$ and declining to $58 \%$ for the past $10 \mathrm{~m} . \mathrm{y}$. Calcium carbonate contents in the deepest site (929) are similar to those in the shallower sites where the records overlap older than $25 \mathrm{Ma}$, and are generally lower than those in the shallower sites in younger sediments, with the largest differences in the 10-25 Ma interval. Site 929 is marked by a hiatus from 8.4 to 9.4 Ma (at 205.90 meters below seafloor). This hiatus is underlain by an interval of low sedimentation rate (Shipboard Scientific Party, 1995d), presumably caused by dissolution. We have one sample from this interval, with a calcium carbonate content of $4.8 \%$, the lowest we

observed. Calcium carbonate sedimentation rates generally decrease with increasing site water depth, and the two northernmost sites have dramatic increases in detrital sedimentation rates in the past 10 m.y., apparently from increased Andean erosion (Shipboard Scientific Party, 1995a).

Total $\mathrm{P}$ concentrations for samples from all three sites range from 8 to $22 \mu \mathrm{mol}$ P/g sediment (Tables 1,2). Total reactive P (i.e., the sum of $\mathrm{P}$ concentrations in the sedimentary components that serve to remove reactive $\mathrm{P}$ from the oceanic reservoir), accounts for the majority of sedimentary $\mathrm{P}$ in all sites and is relatively constant with age (Fig. 3F; Table 1). The low calcium-carbonate-content, low sedimentation-rate sample from Site 929 is an exception to these trends, marked by relatively high authigenic $\mathrm{P}$ concentrations (and by high adsorbed and low oxide-bound $\mathrm{P}$ ) and therefore by relatively high total reactive $\mathrm{P}$ concentrations (Table 1). Detrital P (Fig. 3D; Table 1) 
Table 2. Summary of site characteristics, sample characteristics, and $P$ concentrations.

\begin{tabular}{|c|c|c|c|}
\hline & Site 925 & Site 926 & Site 929 \\
\hline \multicolumn{4}{|l|}{ Site characteristics: } \\
\hline Water depth (m) & 3041 & 3598 & 4356 \\
\hline Latitude $\left({ }^{\circ} \mathrm{N}\right)$ & 4.2 & 3.7 & 6.0 \\
\hline Longitude $\left({ }^{\circ} \mathrm{W}\right)$ & 43.5 & 42.9 & 43.7 \\
\hline Primary productivity & $35-60$ & $35-60$ & $35-60$ \\
\hline \multicolumn{4}{|l|}{ Sample characteristics: } \\
\hline Sample number & 38 & 24 & 30 \\
\hline Depth range (mbsf) & $16-920$ & $15-590$ & $11-783$ \\
\hline Age range $(\mathrm{Ma})$ & $0.5-43$ & $0.5-29$ & $0.3-55$ \\
\hline Sedimentation rate range (mbsf/m.y.) & $10-50$ & $11-29$ & $3-37$ \\
\hline \multicolumn{4}{|l|}{ Calcium carbonate content $(\%)$} \\
\hline $\begin{array}{l}\text { Mean } \pm 2 s / \sqrt{N} \\
\text { (Range) }\end{array}$ & $\begin{array}{l}67 \pm 4 \\
(35-84)\end{array}$ & $\begin{array}{l}65 \pm 4 \\
(48-81)\end{array}$ & $\begin{array}{c}48 \pm 6 \\
(5-71)\end{array}$ \\
\hline \multicolumn{4}{|l|}{$P$ concentration: } \\
\hline \multicolumn{4}{|l|}{ Total } \\
\hline $\begin{array}{l}\text { Mean } \pm 2 \mathrm{~s} / \sqrt{N} \\
\text { (Range) }\end{array}$ & $\begin{array}{c}12.5 \pm 0.5 \\
(8.6-16)\end{array}$ & $\begin{array}{c}10.4 \pm 0.8 \\
(7.7-15)\end{array}$ & $\begin{array}{c}13.3 \pm 1.2 \\
(7.9-22)\end{array}$ \\
\hline \multicolumn{4}{|l|}{ Total reactive } \\
\hline $\begin{array}{l}\text { Mean } \pm 2 \mathrm{~s} / \sqrt{N} \\
\text { (Range) }\end{array}$ & $\begin{array}{r}11.9 \pm 0.5 \\
(8.5-15)\end{array}$ & $\begin{array}{c}10.1 \pm 0.7 \\
(7.4-15)\end{array}$ & $\begin{array}{c}12.7 \pm 1.1 \\
(7.8-21)\end{array}$ \\
\hline \multicolumn{4}{|l|}{ Authigenic } \\
\hline $\begin{array}{l}\text { Mean } \pm 2 \mathrm{~s} / \sqrt{N} \\
\text { (Range) }\end{array}$ & $\begin{array}{l}9.1 \pm 0.7 \\
(4.3-13)\end{array}$ & $\begin{array}{l}7.5 \pm 0.7 \\
(4.9-12)\end{array}$ & $\begin{array}{l}8.8 \pm 0.9 \\
(5.3-16)\end{array}$ \\
\hline Fraction of total reactive $P$ & 0.76 & 0.74 & 0.69 \\
\hline \multicolumn{4}{|l|}{ Organic } \\
\hline $\begin{array}{l}\text { Mean } \pm 2 s / \sqrt{N} \\
\text { (Range) }\end{array}$ & $\begin{array}{l}1.1 \pm 0.2 \\
(0.3-3.5)\end{array}$ & $\begin{array}{l}0.9 \pm 0.2 \\
(0.3-1.7)\end{array}$ & $\begin{array}{l}1.6 \pm 0.4 \\
(0.3-4.2)\end{array}$ \\
\hline Fraction of total reactive $P$ & 0.09 & 0.09 & 0.12 \\
\hline \multicolumn{4}{|l|}{ Oxide-bound } \\
\hline $\begin{array}{l}\text { Mean } \pm 2 \mathrm{~s} / \sqrt{N} \\
\text { (Range) }\end{array}$ & $\begin{array}{l}0.9 \pm 0.2 \\
(0.2-2.8)\end{array}$ & $\begin{array}{l}0.9 \pm 0.4 \\
(0.2-3.8)\end{array}$ & $\begin{array}{l}1.3 \pm 0.3 \\
(0.1-3.3)\end{array}$ \\
\hline Fraction of total reactive $P$ & $\begin{array}{l}(0.2-2.8) \\
0.08\end{array}$ & $\begin{array}{c}(0.2-5.8) \\
0.09\end{array}$ & $\begin{array}{l}(0.1-5.3) \\
0.10\end{array}$ \\
\hline \multicolumn{4}{|l|}{ Adsorbed } \\
\hline $\begin{array}{l}\text { Mean } \pm 2 s / \sqrt{N} \\
\text { (Range) }\end{array}$ & $\begin{array}{c}0.8 \pm 0.2 \\
(0.04-0.23)\end{array}$ & $\begin{array}{l}0.9 \pm 0.2 \\
(0.4-2.4)\end{array}$ & $\begin{array}{l}1.0 \pm 0.3 \\
(0.1-2.7)\end{array}$ \\
\hline Fraction of total reactive $\mathrm{P}$ & $\begin{array}{l}(0.04-0.25) \\
0.07\end{array}$ & 0.09 & 0.08 \\
\hline \multicolumn{4}{|l|}{ Detrital } \\
\hline $\begin{array}{l}\text { Mean } \pm 2 \mathrm{~s} / \sqrt{N} \\
\text { (Range) }\end{array}$ & $\begin{array}{c}0.6 \pm 0.2 \\
(0.07-2.5)\end{array}$ & $\begin{array}{c}0.4 \pm 0.1 \\
(\mathrm{BDL}-0.9)\end{array}$ & $\begin{array}{r}0.7 \pm 0.3 \\
(\mathrm{BDL}-2.8)\end{array}$ \\
\hline
\end{tabular}

Notes: Site characteristics from Shipboard Scientific Party (1995b, 1995c, 1995d). Primary productivity $\left(\mathrm{g} \mathrm{C} \mathrm{m}^{-2} \mathrm{y}^{-1}\right)$ from synthetic primary productivity map of Berger and Wefer (1991). P concentrations measured as $\mu \mathrm{mol} \mathrm{P/g} \mathrm{sediment.} \mathrm{BDL}=$ value below detection limit.

typically accounts for only a few percent of total $\mathrm{P}$, with increased contributions in the past 10 m.y., which is the interval of increasing detrital mass accumulation rates. Total reactive $\mathrm{P}$ concentrations have similar ranges and means for these three sites, with means $\pm 2 \mathrm{~s} /$ $\sqrt{N}$ of $11.9 \pm 0.5,10.1 \pm 0.7$, and $12.7 \pm 1.1 \mu \mathrm{mol} \mathrm{P/g}$ sediment for Sites 925, 926, and 929, respectively (Table 2; Fig. 3F). Authigenic $\mathrm{P}$ (Fig. 3C) is the major sedimentary component of both total and total reactive $P$ at all three sites.

Before about $10 \mathrm{Ma}$, detrital $\mathrm{P}$ concentrations (Fig. 3D; Table 3) are low (less than $3.3 \%$ of total P) and similar at all three sites, typically $0.3-0.4 \mu \mathrm{mol} \mathrm{P} / \mathrm{g}$ sediment. From $10 \mathrm{Ma}$ to the present, detrital $\mathrm{P}$ concentrations increase by a factor of five in the most northerly Site 929 and by a factor of three in Site 925 . Site 926 has no significant change in detrital $\mathrm{P}$ concentration (Table 3 ). These observations are consistent with a dramatic increase in noncarbonate mass accumulation rates observed in the more northern sites (Shipboard Scientific Party, 1995a) and with the increase in detrital quartz at Site 925 in the past 6 m.y., based on Fourier Transform Infrared Spectroscopy records of sediment composition (T. Herbert, pers. comm., 1995).

Adsorbed and oxide-bound $\mathrm{P}$ concentrations generally decrease with increasing age to about $10 \mathrm{Ma}$ (Figs. 3A, 3B). Mean concentrations for these components in sediments up to $10 \mathrm{~m}$.y. old are larger than those for older sediments (Table 3 ). In addition, organic P concentrations (Fig. 3E) decrease with increasing age up to 10-15 Ma in the two shallower sites and to 25-30 Ma in the deepest site. Especially in the 10-25 Ma interval, organic $\mathrm{P}$ concentrations tend to be highest at the deepest site. The mean organic $P$ concentrations for all three sites are relatively low (Tables 2,3 ), consistent with the low organic carbon contents of these sediments (generally $<0.4 \mathrm{wt} \%$; see solidphase geochemistry sections of the respective site chapters).

There are distinctive increases in the concentrations of authigenic P over time, which mirror the decreases in adsorbed, oxide-bound, and organic $\mathrm{P}$ components. From $10 \mathrm{Ma}$ to present, authigenic $\mathrm{P}$ constitutes $32 \%-70 \%$ of total reactive $P$. In contrast, authigenic $P$ constitutes $76 \%-85 \%$ of total reactive $\mathrm{P}$ on average in sediments older than $10 \mathrm{Ma}$ (Table 3).

$\mathrm{P}$ distributions and their trends with sediment age indicate a reorganization of $\mathrm{P}$ from more reactive components, such as those that originally transported $\mathrm{P}$ to the sediment (e.g., organic $\mathrm{P}$ ) or sequestered it during early sedimentary transformations of $\mathrm{P}$ (e.g., adsorbed $\mathrm{P}$, oxide-bound $\mathrm{P}$ ) to authigenic $\mathrm{P}$. This process of "sink-switching" has been documented in continental margin sediments, including those in nonupwelling regions (Ruttenberg and Berner, 1993), and more recently, in open-ocean sediments (Filippelli and Delaney, 1995, 1996).

Because different oceanographic regions and different sites within a given region may have time-dependent trends in total reactive $\mathrm{P}$ concentrations, an informative way of demonstrating the widespread significance of $\mathrm{P}$ reorganization in open-ocean sediments is to consider the fraction of total reactive $\mathrm{P}$ identified as the authigenic component that is a function of sediment age. We find that the trends in the fraction of reactive $\mathrm{P}$ that is present as authigenic $\mathrm{P}$ relative to sediment age are similar for all three Ceara Rise sites (Fig. 4A). This similarity for sites in a given oceanographic regime holds for three eastern equatorial Pacific sites (Fig. 4B) and four western equatorial Pacific sites (Fig. 4C). Across a range of surface water productivity and site water depth (see Table 2 and Fig. 4 caption for detailed information), the fraction of total reactive $\mathrm{P}$ that is present as authigenic $\mathrm{P}$ increases at all sites in all three regions with increasing sediment age until it plateaus. The redistribution of adsorbed, oxide-bound, and organic components to the authigenic component occurs more rapidly in the Pacific sites (within the first 7 m.y.) than the Atlantic (within 20 m.y.). Authigenic $\mathrm{P}$ constitutes the majority of reactive $\mathrm{P}$, accounting for approximately $80 \%$ of total reactive $\mathrm{P}$ in older sediments from the Ceara Rise sites (Fig. 4A) and for approximately $85 \%$ for the eastern equatorial Pacific sites (Fig. 4B) and 90\% for the Ontong Java Plateau sites (Fig. 4C). The relatively small differences in fraction authigenic $\mathrm{P}$ in the older sediments appear to be due to higher proportions of organic and adsorbed $\mathrm{P}$ in older Ceara Rise sediments relative to the equatorial Pacific sediments. The differences between the eastern and western equatorial Pacific sites primarily reflect a larger influence of oxide-bound $\mathrm{P}$ in the eastern equatorial Pacific sites (Filippelli and Delaney, 1995, 1996).

The three sites have generally similar total reactive $\mathrm{P}$ concentrations over a relatively wide range of open-ocean, linear sedimentation rates (Table 2). The use of linear sedimentation rates, representing relatively long-term averages, for evaluating diagenetic redistribution of $\mathrm{P}$ is appropriate because the formation of authigenic $\mathrm{P}$ most likely involves $\mathrm{P}$ transfer by way of interstitial water with accompanying diffusive homogenization. Total reactive $\mathrm{P}$ concentration has no significant correlation with linear sedimentation rate for all sites (Fig. 5A; slope not significantly different from zero using t-test [Dowdy and Weardon, 1983]). This suggests that sedimentation rates do not exert a major control on $\mathrm{P}$ concentrations on these time scales.

The differences in calcium carbonate content from site to site at any one time are driven primarily by increasing calcium carbonate dissolution with increasing water depths, whereas the trends within a given site depend on the balance of carbonate productivity, dilution by noncarbonate material, and dissolution effects. There are two endmember possibilities for the effect of calcium carbonate dissolution on reactive $\mathrm{P}$ burial in sediments: reactive $\mathrm{P}$ is lost from the sediments when calcium carbonate dissolves (the "dissolution" hypothesis) or reactive $\mathrm{P}$ is retained in the sediments despite calcium carbonate dissolution, so that $\mathrm{P}$ mass accumulation rates are accurate reflections of 
A

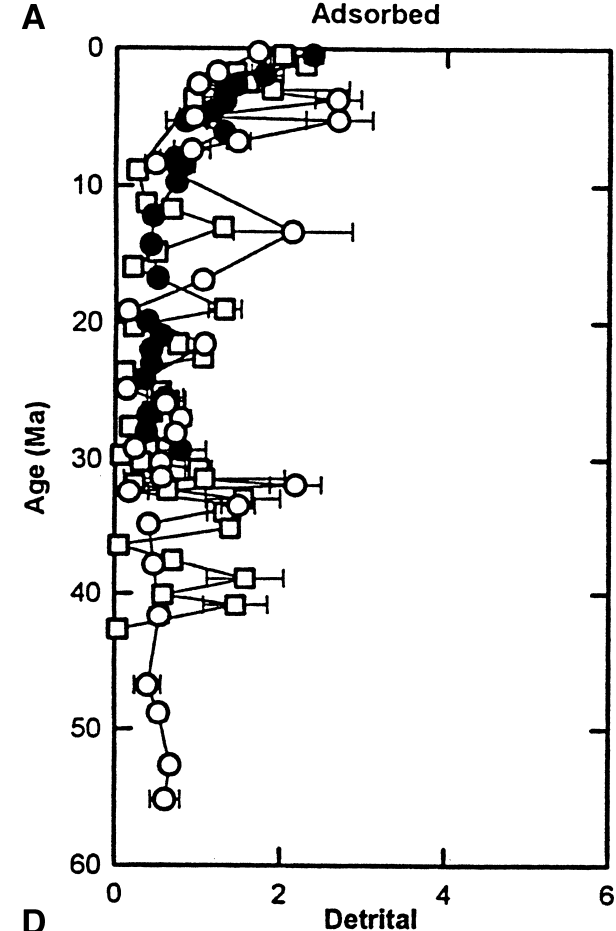

D

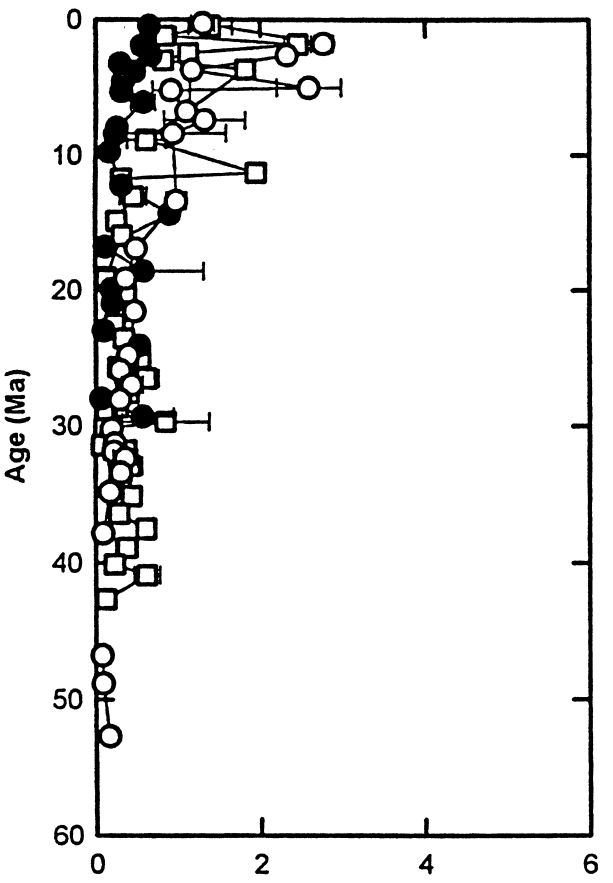

B
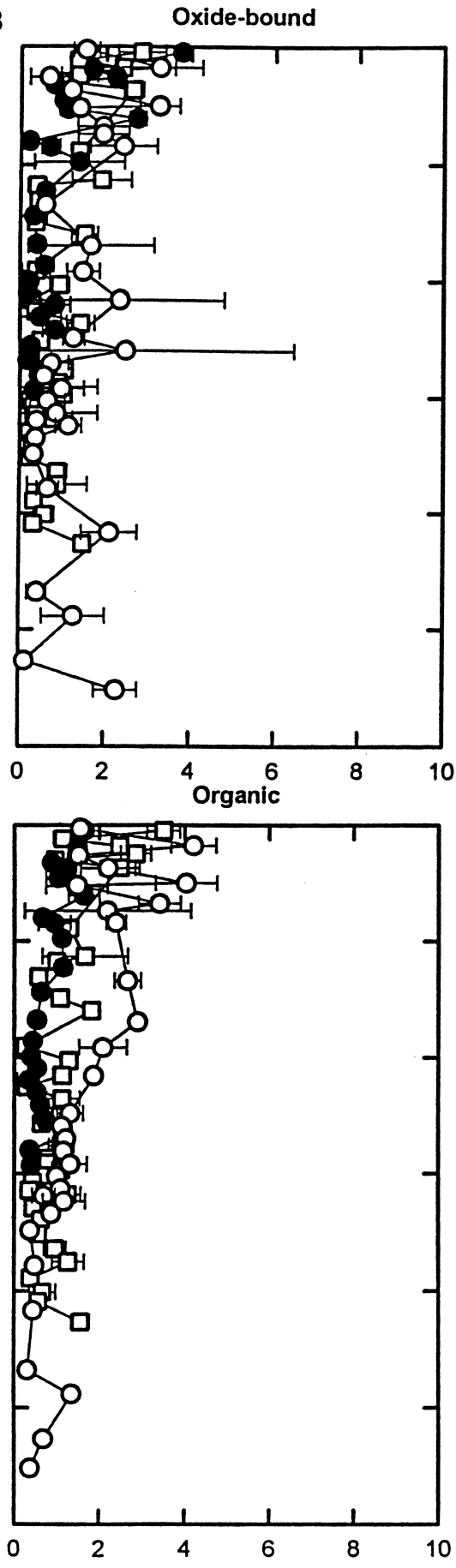

C

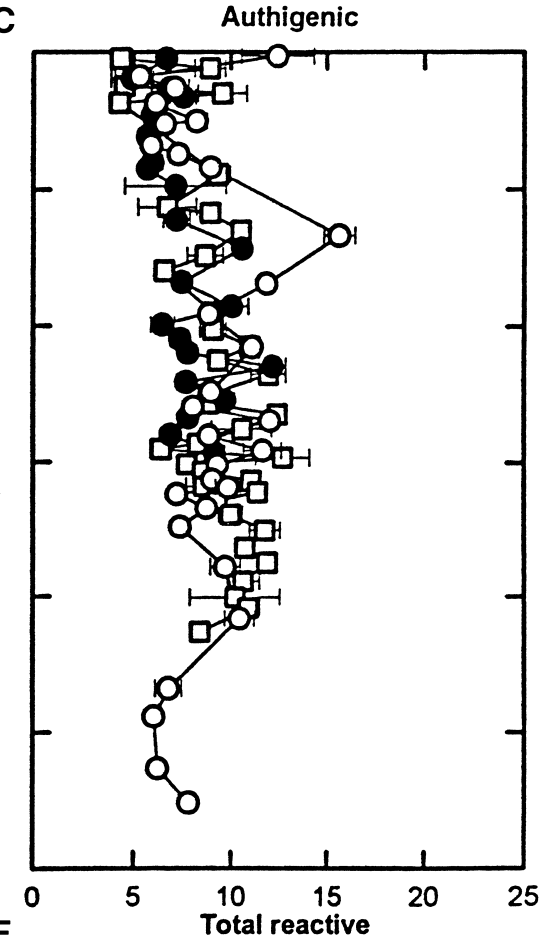

F

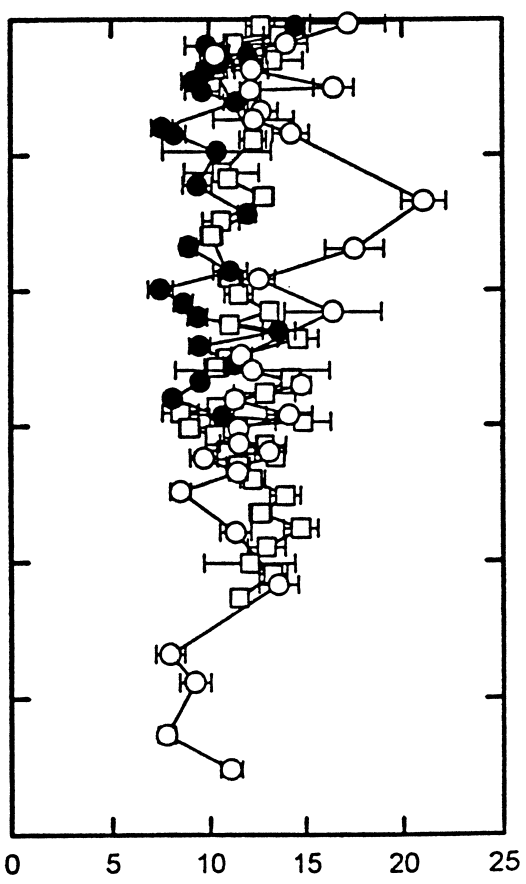

P concentration ( $\mu \mathrm{mol} \mathrm{P} / \mathrm{g}$ sediment)

Figure 3. Age vs. phosphorus concentrations for the five sedimentary components distinguished by sequential extraction and total reactive P for Sites 925 (open squares), 926 (solid circles), and 929 (open circles). Components are presented in order of extraction step. Note that $10 \mu$ mol P/g sediment is equivalent to approximately $0.03 \mathrm{wt} \%$ P. A. Adsorbed P. B. Oxide-bound P. C. Authigenic P. D. Detrital P. E. Organic P. If organic C was being buried with the Redfield ratio of C:P for marine organic matter of 106:1, $1 \mu$ mol organic P/g sediment would be equivalent to an organic carbon content of $0.13 \mathrm{wt} \%$. F. Total reactive P. Note different horizontal scales for the different components. Error bars represent $\pm 1 \mathrm{~s}$ standard deviation on means from replicate determinations.

reactive $\mathrm{P}$ outputs (the "ocean history" hypothesis). The dissolution hypothesis would predict that reactive $\mathrm{P}$ concentrations should decrease as calcium carbonate contents decrease from dissolution effects. The ocean history hypothesis predicts the opposite: reactive $\mathrm{P}$ would be retained while a major sedimentary component was lost, leading to higher reactive $\mathrm{P}$ concentrations with lower calcium car- bonate contents. Although a complete interpretation of sedimentary $\mathrm{P}$ concentrations would have to incorporate such things as changing $\mathrm{P}$ inputs through time, total reactive $\mathrm{P}$ concentration does not decrease as calcium carbonate content decreases (Fig. 5B; see figure caption for regression statistics). The lack of correlation of reactive $\mathrm{P}$ with either linear sedimentation rate or calcium carbonate content 
Table 3. Summary of $P$ concentrations for five sedimentary components in two age windows.

\begin{tabular}{|c|c|c|c|}
\hline & \multicolumn{3}{|c|}{$\mathrm{P}$ concentration $(\mu \mathrm{mol} \mathrm{P} / \mathrm{g}$ sediment $)$} \\
\hline & Site 925 & Site 926 & Site 929 \\
\hline \multicolumn{4}{|l|}{ Total: } \\
\hline$<10 \mathrm{Ma}$ & $13.3 \pm 0.9$ & $10.7 \pm 1.2$ & $15.2 \pm 1.6$ \\
\hline$\geq 10 \mathrm{Ma}$ & $12.3 \pm 0.6$ & $10.3 \pm 1.0$ & $12.6 \pm 1.5$ \\
\hline \multicolumn{4}{|c|}{ Total reactive: } \\
\hline$<10 \mathrm{Ma}$ & $11.9 \pm 1.3$ & $10.3 \pm 1.1$ & $13.5 \pm 1.5$ \\
\hline$\geq 10 \mathrm{Ma}$ & $12.0 \pm 0.5$ & $10.0 \pm 0.9$ & $12.3 \pm 1.4$ \\
\hline \multicolumn{4}{|c|}{ Authigenic: } \\
\hline$<10 \mathrm{Ma}$ & $6.6 \pm 1.9$ & $6.3 \pm 0.5$ & $7.6 \pm 1.4$ \\
\hline$\geq 10 \mathrm{Ma}$ & $9.7 \pm 0.6$ & $8.5 \pm 0.9$ & $9.3 \pm 1.0$ \\
\hline \multicolumn{4}{|l|}{ Organic: } \\
\hline$<10 \mathrm{Ma}$ & $2.1 \pm 0.7$ & $1.2 \pm 0.2$ & $2.6 \pm 0.7$ \\
\hline$\geq 10 \mathrm{Ma}$ & $0.9 \pm 0.2$ & $0.6 \pm 0.2$ & $1.2 \pm 0.3$ \\
\hline \multicolumn{4}{|c|}{ Oxide-bound: } \\
\hline$<10 \mathrm{Ma}$ & $1.8 \pm 0.6$ & $1.5 \pm 0.6$ & $2.0 \pm 0.6$ \\
\hline$\geq 10 \mathrm{Ma}$ & $0.7 \pm 0.2$ & $0.4 \pm 0.1$ & $1.1 \pm 0.3$ \\
\hline \multicolumn{4}{|l|}{ Adsorbed: } \\
\hline$<10 \mathrm{Ma}$ & $1.5 \pm 0.5$ & $1.3 \pm 0.3$ & $1.5 \pm 0.5$ \\
\hline$\geq 10 \mathrm{Ma}$ & $0.7 \pm 0.2$ & $0.5 \pm 0.1$ & $0.7 \pm 0.3$ \\
\hline \multicolumn{4}{|l|}{ Detrital: } \\
\hline$<10 \mathrm{Ma}$ & $1.3 \pm 0.5$ & $0.4 \pm 0.1$ & $1.6 \pm 0.5$ \\
\hline$\geq 10 \mathrm{Ma}$ & $0.4 \pm 0.1$ & $0.4 \pm 0.1$ & $0.3 \pm 0.1$ \\
\hline
\end{tabular}

Note: $\mathrm{P}$ concentrations $=$ means $\pm 2 \mathrm{~s} / \sqrt{N}$ standard deviations in $\mu \mathrm{mol} \mathrm{P} / \mathrm{g}$ sediment.

suggests that diagenetic transformations of reactive $\mathrm{P}$ serve to retain $\mathrm{P}$ in the sediments.

\section{CONCLUSIONS}

The trends of P geochemistry with age for these Ceara Rise sites demonstrate that diagenetic reorganization of $\mathrm{P}$ in sediments is an important influence on $\mathrm{P}$ burial, serving to retain $\mathrm{P}$ in sediments. Authigenic $\mathrm{P}$ is an important sediment component for $\mathrm{P}$ burial at all sites, and the relative importance of this component increases with increasing sediment age. This pattern in these sites is similar to those for sites in the eastern and western equatorial Pacific. Total reactive $P$ appears to be retained in the sediments despite calcium carbonate dissolution. These observations are important in understanding how to interpret $\mathrm{P}$ concentration records as $\mathrm{P}$ accumulation records.

\section{ACKNOWLEDGMENTS}

We thank W.B. Curry and N.J. Shackleton (co-chief scientists), Carl Richter (ODP staff scientist), and the Shipboard Scientific Party of Leg 154 for their generosity in facilitating this shore-based study. We thank Rachele Hoag and Henry Lau for their assistance with laboratory work. Rob Franks and the Marine Analytical Laboratory of the UCSC Institute of Marine Sciences have provided consistent and high-quality support for this work. Support of this work by NSF Grant No. OCE-9416593 to MLD is gratefully acknowledged. We thank Harry Elderfield and Gabe Filippelli for their thoughtful reviews.

\section{REFERENCES}

Berger, W.H., and Wefer, G., 1991. Productivity of the glacial ocean: discussion of the iron hypothesis. Limnol. Oceanogr., 36:1899-1918.

Delaney, M.L., and Filippelli, G.M., 1994. An apparent contradiction in the role of phosphorus in the Cenozoic mass balances for the world ocean. Paleoceanography, 9:513-527.

Dowdy, S., and Wearden, S., 1983. Statistics for Research: New York (Wiley).

Filippelli, G.M., and Delaney, M.L., 1994. The oceanic phosphorus cycle and continental weathering during the Neogene. Paleoceanography, 9:643652.

, 1995. Phosphorus geochemistry and accumulation rates in the eastern equatorial Pacific Ocean: results from Leg 138. In Pisias, N.G., Mayer, L.A., Janecek, T.R., Palmer-Julson, A., and van Andel, T.H. (Eds.), Proc. ODP, Sci. Results, 138: College Station, TX (Ocean Drilling Program), 757-767.

- 1996. Phosphorus geochemistry of equatorial Pacific sediments. Geochim. Cosmochim. Acta., 60:1479-1495.

Kroenke, L.W., Berger, W.H., Janecek, T.R., et al., 1991. Proc. ODP, Init. Repts., 130: College Station, TX (Ocean Drilling Program).

Mayer, L., Pisias, N., Janecek, T., et al., 1992. Proc. ODP, Init. Repts., 138 (Pts. 1 and 2): College Station, TX (Ocean Drilling Program).

Ruttenberg, K.C., 1990. Diagenesis and burial of phosphorus in marine sediments: implications for the marine phosphorus budget [Ph.D. dissert.]. Yale Univ., New Haven, CT.

, 1992. Development of a sequential extraction method for different forms of phosphorus in marine sediments. Limnol. Oceanogr., $37: 1460-1482$.

Ruttenberg, K.C., and Berner, R.A., 1993. Authigenic apatite formation and burial in sediments from non-upwelling, continental margin environments. Geochim. Cosmochim. Acta, 57:991-1007.

Shipboard Scientific Party, 1995a. Leg 154 synthesis. In Curry, W.B., Shackleton, N.J., Richter, C., et al., Proc. ODP, Init. Repts., 154: College Station, TX (Ocean Drilling Program), 421-442.

, 1995b. Site 925. In Curry, W.B., Shackleton, N.J., Richter, C., et al., Proc. ODP, Init. Repts., 154: College Station, TX (Ocean Drilling Program), 55-152.

- 1995c. Site 926. In Curry, W.B., Shackleton, N.J., Richter, C., et al., Proc. ODP, Init. Repts., 154: College Station, TX (Ocean Drilling Program), 153-232.

, 1995d. Site 929. In Curry, W.B., Shackleton, N.J., Richter, C., et al., Proc. ODP, Init. Repts., 154: College Station, TX (Ocean Drilling Program), 337-417.

Strickland, J.D.H., and Parsons, T.R., 1972. A Practical Handbook of Seawater Analysis: Ottawa (Fisheries Research Board of Canada).

Watanabe, F.S., and Olsen, S.R., 1962. Colorimetric determination of phosphorus in water extracts of soils. Soil Sci., 93:183-188.

Date of initial receipt: 1 December 1995

Date of acceptance: 26 March 1996

Ms 154SR-124 
A

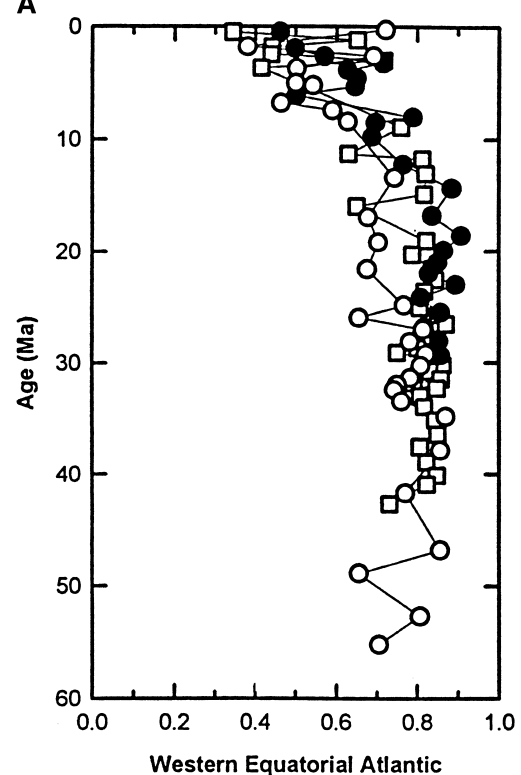

B

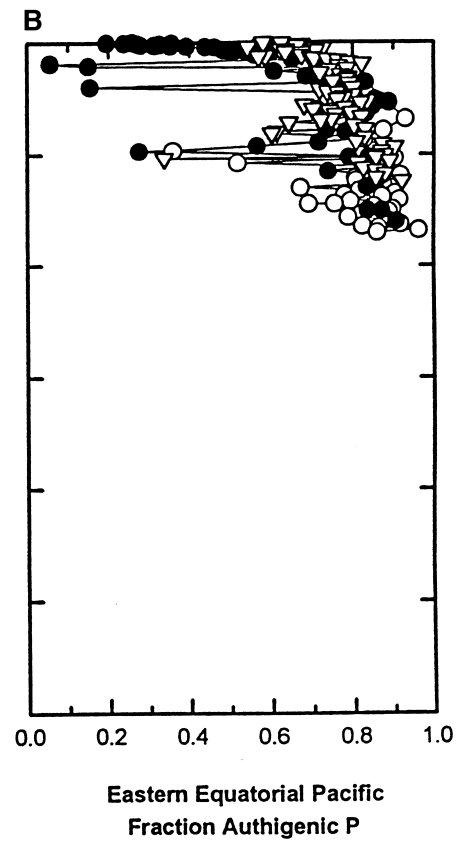

C

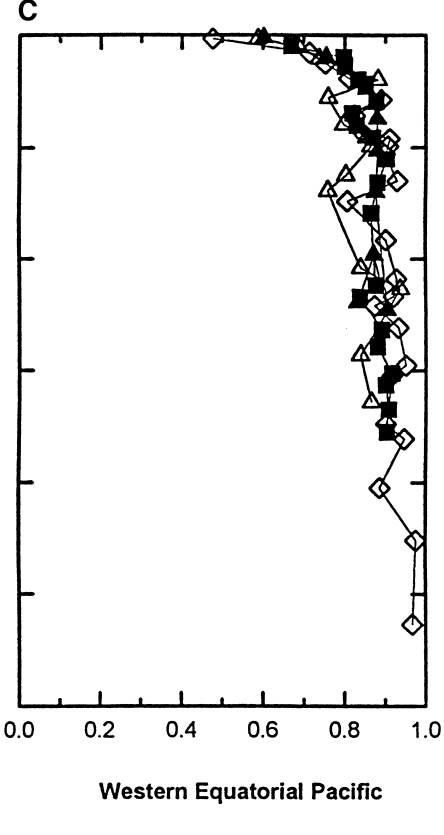

Figure 4. Age vs. fraction authigenic P. A. Leg 154, Ceara Rise Sites 925 (open squares), 926 (solid circles), and 929 (open circles) from this study. B. Leg 138 , eastern equatorial Pacific, Site 851 (open inverted triangles; $3760 \mathrm{~m}$ water depth; mean total reactive $\mathrm{P}= \pm 2 \mathrm{~s} / \sqrt{N}, 10.9 \pm 0.5 \mu$ mol $\mathrm{P} / \mathrm{g}$ sediment $[N=68]$; primary productivity $=100-200 \mathrm{~g} \mathrm{C} \mathrm{m}^{-2} \mathrm{y}^{-1}$ ); Site 844 (open circles; $3415 \mathrm{~m}$ water depth; mean total reactive $\mathrm{P}=11.8 \pm 2.6 \mu \mathrm{mol} \mathrm{P} / \mathrm{g}$ sediment $[N=34]$; primary productivity $=100-200 \mathrm{~g} \mathrm{C} \mathrm{m}^{-2} \mathrm{y}^{-1}$ ); and Site 846 (solid circles; $3296 \mathrm{~m}$ water depth; mean total reactive $\mathrm{P}=13.4 \pm 1.1 \mu$ mol $\mathrm{P} / \mathrm{g}$ sediment $[N=68]$; primary productivity $\left.=60-100 \mathrm{~g} \mathrm{C} \mathrm{m}^{-2} \mathrm{y}^{-1}\right)$. The samples with low fractions of authigenic $\mathrm{P}$ in these sites are dominated by the oxide-bound component, with this variability apparently reflecting varying hydrothermal influence at these sites. C. Leg 130, Ontong-Java Plateau water depth transect, western equatorial Pacific, Site 806 (solid triangles; $2520 \mathrm{~m}$ water depth; mean total reactive $\mathrm{P}=7.5 \pm 1.6 \mu \mathrm{mol} \mathrm{P} / \mathrm{g}$ sediment $[N=9$ ]); Site 807 (open diamonds; $2806 \mathrm{~m}$ water depth; mean total reactive $\mathrm{P}=7.6 \pm 1.1 \mu \mathrm{mol} \mathrm{P} / \mathrm{g}$ sediment $[N=24]$ ); Site 803 (solid squares; $3410 \mathrm{~m}$ water depth; mean total reactive $\mathrm{P}=7.8 \pm 1.1 \mu \mathrm{mol} \mathrm{P} / \mathrm{g}$ sediment $[N=$ 19]); and Site 804 (open triangles; $3861 \mathrm{~m}$ water depth; mean total reactive $\mathrm{P}=10.2 \pm 2.0 \mu \mathrm{mol} \mathrm{P} / \mathrm{g}$ sediment $[N=15]$ ). Primary productivity for all Leg 130 sites is estimated to be in the range of 35 to $60 \mathrm{~g} \mathrm{C} \mathrm{m}^{-2} \mathrm{y}^{-1}$. Figures 4B and 4C are after Filippelli and Delaney (1996). Water depths are from ODP Initial Reports Leg 138 (Mayer, Pisias, Janecek, et al., 1992) and Leg 130 (Kroenke, Berger, Janecek, et al., 1991). Primary productivity ranges are from synthetic primary productivity map by Berger and Wefer (1991).
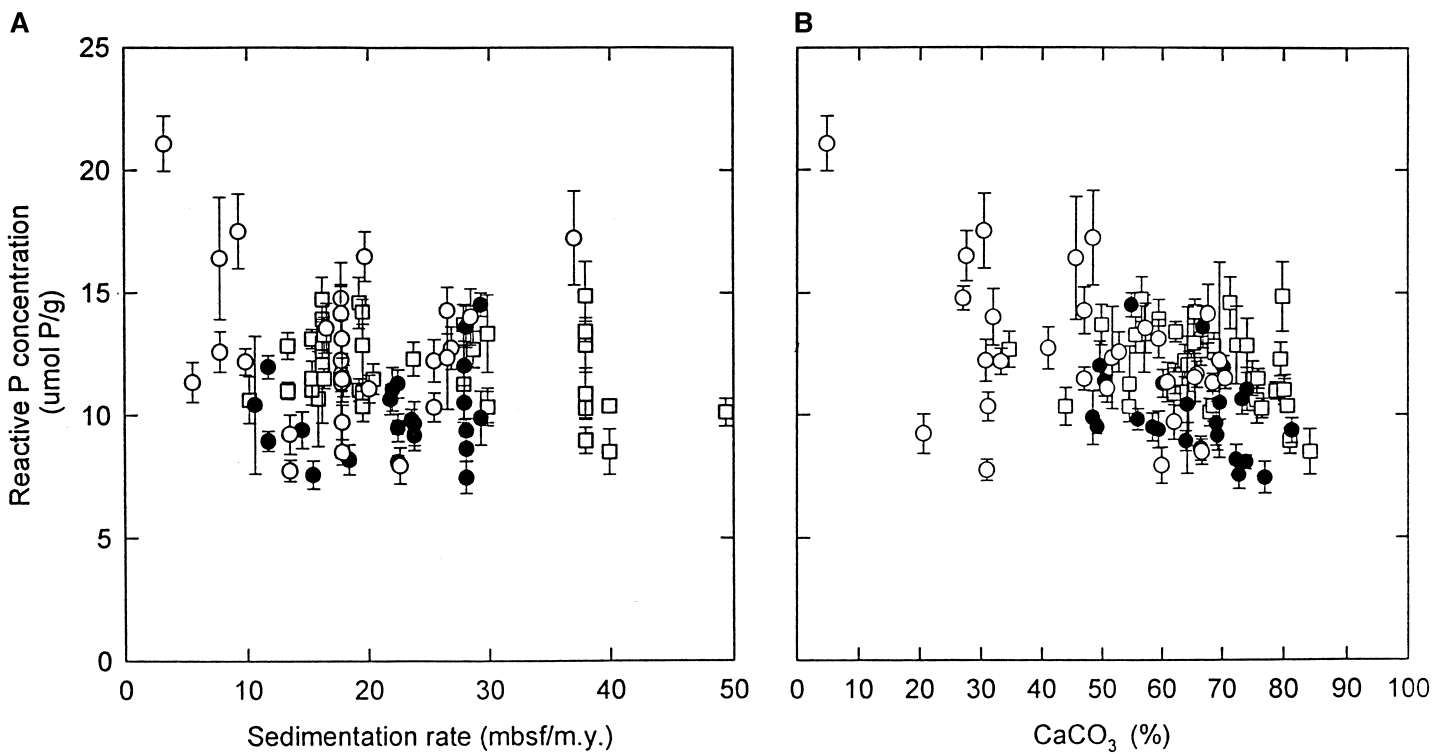

Figure 5. Total reactive $P$ concentrations vs. linear sedimentation rates $(\mathbf{A})$ and calcium carbonate content $(\mathbf{B})$. Site $925=$ open squares, Site $926=$ solid circles, and Site $929=$ open circles. Linear regressions are as follows: Site 925 , intercept $=14.7 \mu \mathrm{mol} \mathrm{P} / \mathrm{g}$ sediment, slope $=-0.042(\mu \mathrm{mol} \mathrm{P} / \mathrm{g}$ sediment $) /(\% \mathrm{CaCO}), r^{2}$ $=0.085, N=38\left(r^{2}\right.$ significant at the $90 \%$ C.L. $)$; Site 926 , intercept $=14.6 \mu \mathrm{mol} \mathrm{P} / \mathrm{g}$ sediment, slope $=-0.070(\mu \mathrm{mol} \mathrm{P} / \mathrm{g} \mathrm{sediment}) /\left(\% \mathrm{CaCO}_{3}\right), r^{2}=0.144, N=$ $24\left(r^{2}\right.$ significant at the $90 \%$ C.L. $)$; Site 929 , intercept $=16.1 \mu \mathrm{mol} \mathrm{P} / \mathrm{g}$ sediment, slope $=-0.072(\mu \mathrm{mol} \mathrm{P} / \mathrm{g}$ sediment $) /\left(\% \mathrm{CaCO}_{3}\right), r^{2}=0.174, N=30\left(r^{2}\right.$ significant at the $95 \%$ C.L. $)$; and all sites, intercept $=15.6 \mu \mathrm{mol} \mathrm{P} / \mathrm{g}$ sediment, slope $=-0.064(\mu \mathrm{mol} \mathrm{P} / \mathrm{g}$ sediment $) /\left(\% \mathrm{CaCO}_{3}\right), r^{2}=0.180, N=92\left(r^{2}\right.$ significant at the 99\% C.L.). C.L. = confidence limit. 